Trans

continentales
Transcontinentales

Sociétés, idéologies, système mondial

$6 \mid 2008$

Mémoires et nations

\title{
Entre l'Inde et le Pakistan
}

Mémoires de la Partition et partition de la mémoire

\section{Lionel Baixas}

\section{OpenEdition}

\section{Journals}

Édition électronique

URL : http://journals.openedition.org/transcontinentales/623

ISBN : 978-2-7351-1561-7

ISSN : 1775-397X

Éditeur

Editions de la maison des sciences de l'homme

\section{Édition imprimée}

Date de publication : 30 juin 2008

Pagination : 73-89

ISBN : 978-2-200-92511-6

ISSN : 1950-1684

Référence électronique

Lionel Baixas, «Entre l'Inde et le Pakistan », Transcontinentales [En ligne], 6 | 2008, document 5, mis en ligne le 06 avril 2011, consulté le 07 septembre 2020. URL : http://journals.openedition.org/

transcontinentales/623 


\title{
Entre I'Inde et le Pakistan
}

\section{Mémoires de la Partition et partition de la mémoire}

\author{
Lionel Baixas
}

\author{
"Ab to tum bhi nahin laut paoge "Maintenant que toi aussi tu as disparu pour toujours \\ Yaad ki sirf ek shart rah jayegi Il ne reste qu'une seule condition pour la mémoire \\ Ki jab bhi kahin Que n'importe quand, n'importe où, \\ Fasad hoga Qu'une émeute se déclenche, \\ Tum bahut yaad aoge." Je me souvienne de toi.» \\ Manazir Aashiq Harganvi
}

Alors que la Partition ${ }^{1}$ de l'Empire britannique des Indes est sans aucun doute l'événement politique et social majeur $\mathrm{du} \mathrm{xx}^{\mathrm{e}}$ siècle dans la région, tant en raison de son rôle fondateur dans la formation des identités nationales indiennes et pakistanaises qu'à cause du traumatisme des violences de la Partition sur les mentalités des populations concernées, il est déroutant de constater qu'il n'existe à ce jour, ni en Inde ni au Pakistan, aucune institutionnalisation de la mémoire de la Partition. Durant les quelques mois qui précédèrent et suivirent l'Indépendance (14 août 1947), près d'un million ${ }^{2}$ de personnes périrent, plus de 15 millions de personnes furent déplacées de part et d'autre des frontières divisant à l'ouest et à l'est l'Inde et le Pakistan - cela représente le plus important transfert de population de l'histoire de l'humanité - et près de 75000 femmes furent enlevées et violées, un chiffre qui les place parmi les premières victimes des violences de la Partition ${ }^{3}$. Pourtant, il n'y a pas eu de commission d'enquête sur les massacres de la Partition, et l'on ne trouve à la frontière indo-pakistanaise ni mémorial ni plaque commémorative

1 - L'emploi de la majuscule, qui se répétera tout au long de l'article, désigne l'événement de la Partition d'août 1947 du Raj britannique, et souligne son caractère fondamental dans l'histoire du sous-continent indo-pakistanais.

2 - Les historiens s'accordent aujourd'hui sur ce nombre d'un million de personnes tuées. Les estimations britanniques officielles de l'époque firent état de 200000 morts, et une estimation indienne plus tardive porta à 2 millions le nombre des victimes.

3 - U. Butalia, The Other Side of Silence: Voices from the Partition of India, New Delhi, Penguin, 1998, p. 3.

Lionel Baixas : Doctorant en sciences politiques, boursier du ministère des Affaires étrangères et européennes, Centre d'études et de recherches internationales (CERI), Sciences Po Paris/Centre de sciences humaines (CSH), New Delhi. 
qui symboliseraient l'expérience et la souffrance des populations ayant subi les violences et l'exode ${ }^{4}$. L'expérience de la Partition est en fait principalement négociée à travers le silence et la stigmatisation de l'autre.

On entend généralement par "partition» la division politique d'un territoire précédemment intégré et des communautés qui le peuplaient. Le terme tend à inclure également, quoique à la marge, les expériences de violence, d'éviction et de migration qui caractérisent en général le processus de division. Mais il désigne bien plus qu'un simple processus de séparation territoriale et la création d'entités politiques distinctes. La partition constitue en effet aussi la matrice pour le développement de concepts et de pratiques de longue durée tels que l'identité nationale, la culture populaire et la mémoire collective, c'est-à-dire les bases mêmes sur lesquelles ces nouvelles sociétés sont instaurées et organisées ${ }^{5}$. La place incertaine et ambigüe qu'occupe la Partition dans les récits historiques indien et pakistanais est liée à la déconnexion qui existe entre l'histoire élitiste de la haute politique de la Partition et l'histoire populaire, par le bas, de l'expérience humaine de la violence de la Partition $^{6}$. Or, la violence ne fit pas qu'accompagner la Partition, elle en est au contraire l'élément constitutif. C'est seulement vers le milieu des années 1940 que la demande pour le Pakistan, apparue comme hypothèse dans la décennie précédente, prit un tour plus concret en se dotant d'une dimension proprement territoriale. Jusqu'à la Cabinet Mission de $1946^{7}$, les négociations constitutionnelles successives au sujet des modalités de transfert du pouvoir des Britanniques vers les leaders politiques indiens envisageaient plutôt la mise en place d'une confédération peu centralisée avec des provinces hindoues et musulmanes autonomes. À la suite de l'échec de la Cabinet Mission, la partition territoriale du Raj britannique devint inéluctable. Dans cette perspective, la violence constitua l'instrument par le biais duquel la tension entre l'identité des communautés religieuses et leur redéfinition en terme territorial fut

4 - Bien au contraire, c'est à une démonstration de patriotisme et de militarisme que se livrent chaque jour au poste-frontière de Wagah-Attari les gardes-frontières indiens et pakistanais, respectivement la Border Security Force (BSF) et les Pakistan Rangers, sous les applaudissements des visiteurs indiens et pakistanais venus assister à la cérémonie de retrait des drapeaux et de clôture des frontières. Voir R. Mc Gill Murphy, «Performing Partition in Lahore», in S. Kaul (dir.), The Partition of Memory : The Afterlife of the Division of India, New Delhi, Permanent Black, 2001, p. 183-208.

5 - S. Tewari Jassal et E. Ben-Ari (dir.), The Partition Motif in Contemporary Conflicts, New Delhi, Sage, 2007, p. 21.

6 - D. Gilmartin, "Partition, Pakistan, and South Asian History: In Search for a Narrative», The Journal of Asian Studies, vol. 54, $\mathrm{n}^{\circ} 4,1998, \mathrm{p} .1069$.

7 - Formée à l'initiative du Premier ministre britannique Clement Attlee, la «Mission du Cabinet» était dirigée par le secrétaire d'État pour l'Inde Lord Frederick Pethick-Lawrence et composée de Sir Stafford Cripps, président de la Chambre du commerce, et de l'amiral Albert Victor Alexander, avec l'aide du vice-roi des Indes, Sir Archibald Wavell; elle fut envoyée en Inde, le 23 mars 1946, afin de poursuivre les négociations concernant les modalités du transfert du pouvoir avec les leaders politiques indiens. La Mission formula deux plans : l'un, daté du 16 mai, préconisait la création d'une confédération indépendante composée de provinces à majorité musulmane et de provinces à majorité hindoue, le gouvernement n'ayant à sa charge que les prérogatives régaliennes traditionnelles; l'autre, daté du 16 juin, recommandait la création de deux États indépendants, l'Inde à majorité hindoue et le Pakistan à majorité musulmane, les États princiers étant autorisés soit à rejoindre l'un ou l'autre, soit à proclamer leur indépendance. L'incapacité et/ou la réticence des leaders du Congrès et de la Ligue musulmane à participer à un gouvernement de coalition rendit la partition territoriale du Raj britannique inéluctable. 
localement négociée. La violence opéra ainsi le lien symbolique entre ces communautés et leur nouveau territoire. C'est précisément cela qui conféra à cette violence sa dimension purificatrice et organisée. C'est par conséquent la Partition, bien plus que l'indépendance vis-à-vis de la Couronne britannique, qui instaura et réifia définitivement la définition territoriale de l'État-nation postcolonial en Asie du Sud ${ }^{8}$.

La tyrannie du silence qui pèse sur la dimension humaine de l'expérience de la Partition empêche toute mémorialisation institutionnelle et collective de l'histoire de cet événement et toute réconciliation entre l'Inde et le Pakistan. Les fantômes de la Partition ont en effet contaminé de nombreux domaines de la vie politique et sociale des deux pays et alimentent tant les conflits intra-étatiques entre les deux frères ennemis, que les conflits nationaux intercommunautaires ou les politiques autoritaires et répressives comme le nationalisme xénophobe et anti-minoritaire. L'irruption de fragments non réconciliés du passé dans le présent contribue ainsi à envenimer la situation en générant un sentiment permanent d'hostilité et d'insécurité. La Partition en tant qu'événement historique ne peut pas être comprise seulement comme un acte qui résulta de l'opération des dynamiques de l'histoire, mais aussi et surtout comme un événement continuellement rappelé à la vie par le jeu de mémoires subjectives ${ }^{9}$, en particulier à travers la transmission intergénérationnelle de récits individuels au sein des familles indiennes et pakistanaises qui l'ont vécue. Par conséquent, la Partition ne devrait pas être étudiée comme un événement fini mais comme un processus qui débuta bien avant 1947 et qui se poursuit encore de nos jours. C'est pour cette raison qu'il importe d'explorer la présence du passé, celle-ci ne devant pas être conçue seulement comme un mode spécifique de représentation du passé, car au-delà de l'événement passé en tant que tel, le fait historique réel et significatif que cette présence illustre est la mémoire ellemême $e^{10}$. Autrement dit, cette mémoire est à la fois un mode de représentation du passé et une réponse à la présence du passé. Certains affirment qu'il est nécessaire de distinguer clairement la mémoire de l'histoire dans la mesure où « une mémoire traumatisée détient une structure narrative qui opère selon un principe opposé à celui de tout récit historique [...] un récit historique nous mènerait jusqu'à l'événement, expliquant pourquoi il est arrivé et pourquoi il est arrivé à ce moment-là [...] ce qui ne peut pas être expliqué normalement appartient à la marginalité de l'histoire $^{11}$ ". Il nous semble au contraire que malgré leurs différences, il existe entre les problèmes internes à l'histoire et les demandes de mémoire une relation remarquablement complémentaire, à tel point qu'elles deviennent parties intégrantes d'une seule et même opération, l'opération historiographique ${ }^{12}$. En effet, "[l]es mémoires des violences se construisent dans l'enchevêtrement entre mémoires individuelles

\footnotetext{
8 - D. Gilmartin, "Partition, Pakistan, and South Asian History...», art. cit., 1998, p. 1089.

9 - R. Samaddar, «The Historiographical Operation : Memory and History», Economic and Political Weekly, 3 juin 2006, p. 2236.

10 - K. Birth, "The Immanent Past : Culture and Psyche at the Juncture of Memory and History", Ethos, vol. 34, $\mathrm{n}^{\circ} 2$, 2006, p. 169-191.

11 - D. Chakrabarty, "Remembered Villages : Representations of Hindu-Bengali Memories in the Aftermath of Partition", in M. Hasan (dir.), Inventing Boundaries: Gender, Politics and the Partition of India, New Delhi, Oxford University Press, 2002, p. 319-320.

12 - R. Samaddar, «The Historiographical Operation...», art. cit., 2006, p. 2238.
} 
et mémoires collectives qui viennent réécrire des mémoires plus lointaines [...]. Les récits qui en résultent doivent être compris à l'intersection de l'histoire collective et de l'histoire psychique, des histoires singulières et des liens de groupe, des liens de groupe et du travail de culture ${ }^{13} »$.

Cette communication entend dès lors étudier les manières divergentes dont le sens et la mémoire de la Partition ont été constitués et reconstitués en Inde et au Pakistan afin d'élucider quel impact les différentes façons de se souvenir et d'écrire sur 1947 ont sur le processus de construction de l'événement et de l'héritage que l'on appelle la Partition. Cela devrait nous permettre de déterminer dans quelle mesure et à quelles conditions il est possible de mémoriser des violences et d'apaiser des traumatismes comme la Partition, ainsi que la nature des enjeux d'une telle mémoire pour les populations comme pour les gouvernements. Comment une société négociet-elle un passé violent? Comment récupère-t-elle d'un traumatisme historique? Comment débute et se développe le processus de guérison et de réconciliation? Quels types d'enjeux doivent être abordés pour que ce processus réussisse?

Nous verrons d'abord comment l'histoire de la dimension humaine de la Partition a été éludée entre 1947 et les années 1990 et quelles en ont été les conséquences. Puis, nous examinerons la façon dont cette histoire a finalement été réhabilitée à partir du milieu des années 1990. Nous montrerons ensuite que la tradition indienne de rejet du passé douloureux et de méfiance à l'égard de l'histoire a facilité le déni initial et continue de représenter un obstacle à la commémoration de la Partition. Finalement, nous présenterons deux initiatives de travail de mémoire sur la Partition que nous avons suivies lors d'une mission de recherche au Pakistan à l'occasion du soixantième anniversaire de l'Indépendance et de la Partition, le 14 août 2007.

\section{L'histoire "orthodoxe» de la Partition ou comment éluder la réalité de la Partition}

Entre 1947 et le milieu des années 1990, l'histoire académique comme l'histoire scolaire ont, par différents moyens, éludé la question de la mémoire des violences et de la dimension humaine de la Partition, contribuant ainsi à faire de celle-ci un événement vide sans histoire propre, et reléguant du même coup dans le néant les histoires individuelles et collectives des personnes et des groupes touchés par la partition, les forçant presque à l'amnésie. Cela permit aux gouvernements indien et pakistanais de l'instrumentaliser afin de promouvoir leur définition des identités nationales respectives de l'Inde et du Pakistan. Alors que «l'héritage psychologique de la Partition a laissé de bien plus profondes cicatrices dans l'esprit des populations que les dynamiques sociales, économiques et politiques qui ont conduit à la

13 - B. Pouligny, "Mémoires et violences : vivre aujourd'hui, imaginer demain », communication à la conférence internationale Mémoire et violence politique dans le monde contemporain, organisée conjointement par l'Institut d'études péruviennes, la Red para el desarrollo de las ciencias sociales, l'Institut français d'études andines et l'ambassade de France au Pérou, Lima, novembre 2002, disponible sur http://www.ceri-sciences-po.org/cherlist/pouligny/Violenciamemoria.pdf, p. 1. 
division ${ }^{14}$ ", ce sont ces dernières qui ont retenu l'attention de l'histoire académique de cet événement. Une histoire confinée à l'étude de la "haute politique» des années 1940, c'est-à-dire à l'étude des tentatives successives de négociations constitutionnelles entre les élites britanniques et celles du Congrès et de la Ligue musulmane, sur la forme politico-territoriale à donner au sous-continent après le retrait du colonisateur britannique ${ }^{15}$. Or, le fait de se concentrer sur les causes, considérées comme étant seules l'apanage de l'analyse historique, et non sur la nature même de l'événement, relégué a priori dans l'ahistorique, a contribué à évincer toute analyse scientifique de la dimension humaine de la Partition ${ }^{16}$. L'histoire de la Partition se retrouve ainsi associée soit à l'histoire de la fin du Raj britannique par les historiens anglo-saxons, soit à l'histoire de l'émergence des États-nations indien et pakistanais par les historiens nationalistes, mais elle n'est jamais étudiée en tant que telle. Malgré tout ce qui les oppose, les historiographies colonialistes et nationalistes ont en commun de marginaliser l'histoire de la Partition et de condamner les violences populaires. Elles considèrent la première comme une anomalie qui serait venue perturber, pour une durée limitée, la trajectoire du sous-continent vers la modernité politique, et les secondes comme une aberration pré-moderne attribuée à l'autre, qu'il serait futile et inutile d'étudier. Une telle violence, en raison de son caractère éminemment singulier et unique, constituerait un "cas limite» qui serait impropre à l'analyse historique. Son analyse scientifique a été disqualifiée d'avance, et l'étude historiographique de la Partition s'est vue dissociée de celle des violences qui l'ont constituée, ces dernières se retrouvant ainsi absentes, sans histoire.

Cet espace vide fut rapidement investi par les gouvernements indien et pakistanais qui y virent l'occasion de promouvoir leurs versions respectives de l'histoire et de l'identité nationale de leur État. La Partition se vit d'emblée attribuer des significations divergentes. Erreur tragique qui ternit le bon déroulement du mouvement d'indépendance nationale et trahison de la cause nationale par une minorité religieuse séparatiste qui entacha la célébration d'une indépendance chèrement acquise pour les Indiens, la Partition est au contraire considérée par les Pakistanais comme le prix, certes douloureux mais nécessaire, à payer pour l'obtention de leur propre indépendance et d'un État-nation pour la communauté musulmane du sous-continent. L'Inde opposa un nationalisme séculariste, inclusif et composite à la théorie des deux nations, selon laquelle les communautés hindoues et musulmanes constituent deux nations distinctes dont chacune requiert un État propre afin de garantir leur liberté et où l'Islam joue le rôle de ciment de la communauté nationale pour les musulmans ${ }^{17}$. On constate néanmoins que même si les historiographies officielles indienne et pakistanaise sont en grande partie constituées par leur contradiction, elles partagent le même objectif : créer un panthéon national destiné à inculquer aux

14 - A. Jalal, "Secularists, Subalterns and the Stigma of "Communalism" : Partition Historiography Revisited», Modern Asian Studies, vol. 30, n³, p. 681.

15 - The Partition Omnibus, Oxford, Oxford University Press, 2006.

16 - G. Pandey, Remembering Partition: Violence, Nationalism and History in India, Cambridge, Cambridge University Press, 2001, p. 49.

17 - P. Hoodboy et A. H. Nayyar, "Rewriting the History of Pakistan», in A. Khan (dir.), Islam, Politics and the State: The Pakistan Experience, London, Zed Books, 1985, p. 164-177. 
citoyens le respect de leur patrie au détriment de l'autre. L'histoire scolaire constitua le principal outil de cette instrumentalisation. En Inde, une autre version de l'historiographie indienne, l'Hindutva, mise en avant par le Sangh Parivar, se fait le pendant de la version pakistanaise en raison de sa nature communaliste et exclusive ${ }^{18}$. Elle repose également sur des distorsions, des mensonges, des exagérations et la diabolisation des communautés minoritaires. Cette politisation de l'histoire officielle, qui résulte de la relation ambiguë entre l'histoire et l'identité nationale et qui s'effectua par le biais de l'invention d'une mémoire collective fictive enseignée à l'école, a largement contribué à perpétuer les tensions et les incompréhensions entre les deux pays. Les instances de falsification et de manipulation au sein des historiographies officielles et nationales de l'Inde et du Pakistan, qui sont à la base de leur tentative de construction de leurs identités nationales respectives, sont ainsi monnaie courante. L'enseignement de l'histoire ne répond pas à des exigences pédagogiques mais bien à des visées idéologiques. Le but n'est pas d'offrir à l'enfant une version objective de l'histoire mais de lui donner une vision glorieuse du passé de son pays et une image négative de l'autre ${ }^{19}$. Les manuels d'histoire sont ainsi truffés de falsifications, comme en témoignent les passages suivants tirés de manuels pakistanais : "Après la Partition du sous-continent, les hindous et les sikhs entamèrent une campagne planifiée avec précision d'exploitation des musulmans à travers toute l'Inde, et en particulier au Pakistan oriental, au cours de laquelle les ennemis hindous et sikhs de l'humanité tuèrent et déshonorèrent des milliers, non des centaines de milliers de femmes, d'enfants, de personnes âgées et de jeunes gens avec une extrême cruauté ${ }^{20}$.» Ou encore: "Tandis que les musulmans procurèrent tout type d'aide à ceux qui désiraient quitter le Pakistan, les populations de l'Inde commirent les pires atrocités à l'encontre des réfugiés musulmans. Ils attaquèrent, pillèrent et massacrèrent les réfugiés qui étaient transportés en bus, en camions ou en trains ${ }^{21}$. " Le mouvement Hindutva en Inde n'est pas en reste lorsqu'il s'agit de manipuler et de falsifier l'histoire afin de diaboliser non seulement le Pakistan mais aussi les musulmans indiens. Toutefois, en Inde, après la défaite du Bharatiya Janata Party en 2004, un nouveau

18 - Le Sangh Parivar est une «famille» d'organisations à caractère social (Rashtriya Swayamsevak Sangh, RSS ou l'Organisation des volontaires nationaux), religieux (Vishwa Hindu Parishad, VHP ou Conseil hindou mondial; Bajrang Dal ou l'Armée d'Hanuman) ou politique (Bharatiya Janata Party, BJP ou Parti du peuple indien), qui cherchent à promouvoir l'Hindutva (littéralement hindouité), un concept formulé par V. D. Sarvakar, en tant qu'idéologie nationale. Ce type d'idéologie est désigné par le concept générique de communalisme, qui signale, dans une perspective péjorative, une stratégie politique fondée sur la fabrication d'une représentation stéréotypée de l'autre à travers une reconstruction sociale et historique des identités, et qui se traduit par des discriminations, une marginalisation sociale et économique, ainsi que de violentes émeutes. Pour des informations générales, consulter les deux recueils de textes compilés par Christophe Jaffrelot, The Sangh Parivar : A Reader, Delhi, Oxford University Press, 2005 et Hindu Nationalism : A Reader, Princeton, Princeton University Press, 2007.

19 - K. Kumar, Prejudice and Pride: School Histories of the Freedom Struggle in India and Pakistan, New Delhi, Viking, 2001, p. 15-28. Voir également K. Hasanain et A. H. Nayyar, "Conflict and Violence in the Educational Process", in Z. Mian et I. Ahmad (dir.), Making Enemies, Creating Conflict : Pakistan's Crises of State and Society, Lahore, Mashal, 1997.

20 - K. K. Aziz, The Murder of History in Pakistan, Lahore, Vanguard, 1993, p. 42.

21 - A. H. Nayyar et Ahmad Salim (dir.), The Subtle Subversion: The State of Curricula and Textbooks in Pakistan, Islamabad, Sustainable Development Policy Institute, 2006, p. 71. 
manuel de sciences politiques destiné aux classes XII a été publié par le NCERT ${ }^{22}$. Ce manuel traite de la question des violences de la Partition de manière objective, en mobilisant notamment des documents littéraires et cinématographiques. C'est une première, mais sa publication a suscité de vives contestations chez les partisans du BJP à l'Assemblée nationale, ce qui démontre combien ce changement est fragile ${ }^{23}$.

Le phénomène des violences de la Partition a été discursivement externalisé, c'està-dire imputé aux "autres » ${ }^{24}$, les Pakistanais pour les Indiens et vice-versa. Cela a entraîné des conséquences désastreuses pour les relations interétatiques entre l'Inde et le Pakistan, qui se sont affrontés dans quatre guerres depuis 1947, principalement autour du Cachemire. Les frontières nationales qui séparent l'Inde et le Pakistan sont, comme nous l'avons déjà noté, des constructions politiques, des projections imaginaires marquant les limites territoriales de la souveraineté. Ainsi, dans l'imaginaire des Indiens et des Pakistanais, l'appartenance nationale est davantage fondée sur des métaphores abstraites du sol, du territoire, de la terre et de l'espace, que sur les notions civiques concrètes de citoyenneté et de représentation politique. Une telle conception de l'identité nationale a ainsi généré une certaine anxiété liée à la crainte de la porosité des frontières, de l'afflux de réfugiés et d'immigrants, des pratiques subversives d'agents de renseignements ou des attentats transfrontaliers perpétrés par des terroristes. En ce qui concerne la politique intérieure, cette conception a entraîné la marginalisation par les gouvernements des groupes minoritaires, en particulier religieux ${ }^{25}$. En Inde, le musulman de nationalité indienne est ainsi stigmatisé, par les

22 - Les étudiants en classe XII ont entre 16 et 17 ans. Il s'agit de la dernière année de l'école secondaire supérieure dans le système éducatif anglo-saxon. Le Conseil national de la recherche et de la formation éducatives (National Council of Educational Research and Training, NCERT) est une agence de moyens, créée par le gouvernement central indien avec son siège à New Delhi, qui assiste et conseille le gouvernement central et les gouvernements des États sur les questions académiques liées à l'éducation scolaire, et qui publie des manuels scolaires utilisés par les écoles publiques et privées qui suivent le programme du Bureau central de l'éducation secondaire (Central Board of Secondary Education, CBSE).

23 - Le NCERT a été au centre d'une intense controverse durant le mandat de l'Alliance démocratique nationale (National Democratic Alliance, NDA) dirigée par le BJP, à la tête du gouvernement central entre 1999 et 2004, en raison de la suppression et de l'ajout de certains passages dans le programme d'histoire des classes du secondaire visant glorifier le passé de l'Inde et sa culture. Voir Irfan Habib, Suvira Jaiswal, et Aditya Mukherjee, History in the New NCERT Textbooks : A Report and an Index of Errors, Calcutta, Indian History Congress, 2003. Certains historiens et médias ont accusés le BJP de promouvoir une «safranisation» (le safran étant la couleur des nationalistes hindous), ou communalisation, de l'enseignement de l'histoire. Consulter Mridula et Aditya Mukherjee, "Communalising Education", Mainstream, 2001. L'Alliance démocratique nationale perdit les élections générales de 2004 et le nouveau gouvernement central formé par l'Alliance unie pour le progrès (United Progressive Alliance, UPA), dirigée par le Congrès, ordonna la publication de nouveaux manuels afin de restaurer le caractère séculariste de l'éducation. Ainsi du manuel de science politique de classe XII, dont la publication généra un tollé à l'Assemblée nationale, à l'instigation du BJP qui accusait ses auteurs d'être des marxistes et des traîtres à la nation. Voir "Education : NCERT Textbooks", Economic and Political Weekly, vol 41, $\mathrm{n}^{\circ} 34,26$ août- ${ }^{\mathrm{er}}$ septembre 2006, p. 3628.

24 - G. Pandey, "The Prose of Otherness», in D. Arnold et D. Hardiman (dir.), Subaltern Studies VIII : Essays in Honour of Ranajit Guha, New Delhi, Oxford University Press, 1994, p. 198-203.

25 - M. Hasan, Legacy of a Divided Nation: India's Muslims since Independence, Boulder, Westview Press, 1997. Voir aussi K. Sinha-Kerkhoff, "Partition Memories, "Minoritization" and Discourses of Rootedness in Jharkand : A Comparison of Cross Border Displaced and "Invisible Refugees" in Ranchi District», communication à la conférence internationale Displaced People in South Asia, Madras Institute of Development Studies, Chennai, 2-4 mars 2001, disponible sur http://www.sephis.org/pdf/sinha.pdf. 
organisations appartenant à la nébuleuse du nationalisme hindou, en tant que traître en puissance ou comme espion à la solde du Pakistan, et les enclaves musulmanes sur le territoire indien sont péjorativement désignées par le terme small Pakistans ${ }^{26}$.

\section{L'histoire «revendiquée» de la Partition ou comment réhabiliter la dimension humaine de la Partition}

Ce n'est qu'à partir du milieu des années 1990, près de cinquante ans après les événements, que les violences et la dimension humaine de la Partition ont commencé à faire l'objet d'une analyse scientifique minutieuse. L'Inde et le Pakistan furent confrontés, au tournant des années 1980 et 1990, respectivement à la résurgence du nationalisme hindou, des discours anti-minoritaires et des violences communautaires comme en témoignent les pogroms anti-sikhs de Delhi en 1984 ou les émeutes anti-musulmans à Bombay en 1992, et à l'essor des conflits intercommunautaires et sectaires, qu'illustrent les conflits ethniques à Karachi et les conflits entre sunnites et chiites au Pendjab pakistanais et dans le nord du pays. Cette polarisation des sociétés indiennes et pakistanaises joua un rôle primordial dans l'activation du processus de réévaluation de l'historiographie de la Partition. En outre, le grand âge de la plupart des survivants de la Partition - entre 70 et 80 ans - généra un sentiment d'urgence qui poussa les chercheurs, les journalistes et les militants de diverses organisations à recueillir leurs témoignages. L'émergence d'une "histoire contestée» de la Partition centrée sur l'expérience des minorités agissantes, qui place l'étude des violences commises au cœur de ses réflexions, se fit essentiellement par le biais du recueil de témoignages et par la mobilisation de ressources littéraires et cinématographiques, dont l'usage, s'il est louable, peut parfois se révéler problématique. L'historien subalterniste Gyanendra Pandey joua un rôle moteur pour promouvoir les violences de la Partition en tant qu'objet historique à part entière ${ }^{27}$. Selon lui, le cœur de la Partition est bien constitué par les violences qui se sont déroulées et qui ont affecté des millions de personnes, et c'est donc ce qu'il importe d'étudier en priorité. S'opposant tant aux historiens de l'école de Cambridge ${ }^{28}$ qu'à ceux $^{\prime}$

26 - La référence à la Partition et au rôle supposé des musulmans dans les atrocités commises est explicite dans les discours de Bal Thackeray - le leader du Shiv Sena (l’Armée de Shivaji), parti régionaliste marathi/hindou fermement implanté dans l'État du Maharastra -, qui accompagnèrent et encouragèrent les émeutes anti-musulmans de décembre 1992 à la suite de la démolition de la Babri Masjid. En témoigne cet éditorial dans Saamna, l'organe de presse en marathi du Shiv Sena dont Thackeray est l'éditeur en chef, daté du 5 décembre 1992, un jour avant la démolition de la mosquée, où il accuse les musulmans indiens d'être des «traîtres qui ont divisé le pays et qui ne nous ont pas laissé respirer tranquille depuis».

27 - L'école de pensée subalterniste (en référence au concept énoncé par Antonio Gramsci) a pour objectif de formuler un nouveau récit historiographique des trajectoires coloniales et postcoloniales de l'Inde et de l'Asie du Sud à partir d'une histoire par le bas - à l'opposé de l'élitisme de l'histoire académique -, se concentrant sur les expériences des gouvernés et des colonisés qui sont considérés comme les principaux agents du changement politique et social. On compte, parmi ses principaux membres : Ranajit Guha, Parta Chatterjee, Gyanendra Pandey, Gayatri Chakravorty Spivak, Gyanendra Prakash, etc.

28 - La formule "école de Cambridge», dans ce contexte, évoque les historiens britanniques qui ont publié, entre 1922 et 1937, les nombreux volumes de la Cambridge History of India, qui fit longtemps autorité, mais qui fut critiquée aussi bien par les historiens indiens nationalistes que par les historiens indiens "postcoloniaux». (NDLR) 
des écoles nationalistes et révisionnistes ${ }^{29}$, il développe une approche fragmentaire par le bas visant à restituer les histoires des autres, les marginalisés qui n'ont jamais eu voix au chapitre ${ }^{30}$. Conscient que l'archive est source de pouvoir, il refuse de se concentrer seulement sur les archives officielles car elles ne reflètent que le point de vue de l'État et pas celui des populations subalternes. Il rejette l'histoire officielle en raison de son souci de normaliser et d'homogénéiser l'histoire nationale. Il s'offusque également de l'attribution des violences à l'autre, le goonda (bandit), le musulman, le sauvage. Il propose au contraire de restaurer la centralité de l'autre et de déconstruire le discours de l'État sur ce dernier pour en neutraliser les effets néfastes sur la condition des minorités. Si Pandey admet l'impossibilité de produire un récit historique objectif et exhaustif de la violence, il suggère toutefois une approche fragmentaire qui, en mettant l'accent sur les communautés minoritaires et non sur la perspective dominante centrée sur l'État et ses archives, permet de s'approcher de la réalité de la violence par le biais de la mise en confrontation de plusieurs registres de discours émanant de multiples acteurs. Cette perspective subalterniste a ainsi ouvert plusieurs nouveaux champs de recherche sur la dimension humaine de la Partition, comme l'étude de la violence et de ses formes ${ }^{31}$, le sort des femmes ${ }^{32}$, l'expérience de déracinement et de réhabilitation des réfugiés ${ }^{33}$ et l'impact sur la diaspora ${ }^{34}$. Le principal

29 - L'école de pensée révisionniste à l'égard de la Partition en Asie du Sud a remis en cause les deux principaux mythes de l'histoire orthodoxe de la haute politique de la Partition, selon lesquels la Ligue musulmane aurait désiré la Partition alors que le Congrès aurait voulu une Inde unie. Les tenants de cette école dissocient donc la Résolution de Lahore de 1940 de la demande pour la création du Pakistan (le terme «Pakistan» n'apparaît d'ailleurs à aucun moment dans le texte de la résolution), et considèrent la position de Jinnah à l'égard de cette demande comme une manœuvre tactique et un argument de négociation visant à maximiser les intérêts de la communauté musulmane au sein d'une structure confédérale, mais en aucun cas comme une volonté de diviser le pays (voir Ayesha Jalal, The Sole Spokesman : Jinnah, the Muslim League and the Demand for Pakistan, Cambridge, Cambridge University Press, 1985). D'autre part, ils critiquent l'engagement du Congrès pour l'unité de l'Inde, dans la mesure où le leadership de ce parti aurait tout fait pour saper le pouvoir de Jinnah, notamment dans le cadre de la Mission du Cabinet. Pour une discussion de l'approche révisionniste, consulter Asim Roy, «Review: The High Politics of India's Partition : The Revisionist Perspective», Modern Asian Studies, vol. 24, n² 2, 1990, p. 385-408.

30 - L'approche fragmentaire développée par Pandey repose sur la mobilisation et l'analyse de «fragments» tels que le journal intime d'un tisserand, le recueil de poèmes d'un poète inconnu, les mythes locaux, les chansons traditionnelles en langues vernaculaires, etc. Ces «fragments " permettraient à l'historien de rédiger une histoire différente de l'histoire orthodoxe centrée sur l'État et ses archives, en faisant émerger d'autres histoires et de nouveaux espaces de réflexions. G. Pandey, «In Defense of the Fragment : Writing about Hindu-Muslim Riots in India Today», Representations, n ${ }^{\circ} 37,1992$, p. 50.

31 - Entre autres, G. Pandey, Remembering Partition..., op. cit., 2001; P. Brass, «The Partition of India and Retributive Genocide in Punjab 1946-47 : Means, Methods, and Purposes», in P. Brass, Forms of Collective Violence : Riots, Pogroms, and Genocide in Modern India, Gurgaon, Three Essays Collective, 2006; et B. A. Hansen, Partition and Genocide: Manifestation of Violence in Punjab, 1937-47, Delhi, India Research Press, 2002.

32 - Par exemple, U. Butalia, The Other Side of Silence..., op. cit., 1998; R. Menon et K. Bhasin, Borders and Boundaries: Women in India's Partition, Delhi, Kali for Women, 1998; et J. Didur, Unsettling Partition: Literature, Gender, Memory, Toronto, Toronto University Press, 2007.

33 - Voir S. Ansari, Life after Partition : Migration, Community and Strife in Sindh 1947-62, Karachi, Oxford University Press, 2006; I. Talbot, Divided Cities: Partition and its Aftermath in Lahore and Amritsar 1947-1957, Karachi, Oxford University Press, 2006; et R. Kaur, Since 1947 : Partition Narratives among Punjabi Migrants of Delhi, Delhi, Oxford University Press, 2007.

34 - Consulter H. Khalique et R. Kohli, Unfinished Histories : Stories of Separation and Belonging from the South Asian Diaspora, Islamabad, Alhmara, 2002; et P. Gosh, Partition and the South Asian Diaspora: Extending the Subcontinent, Delhi, Routledge, 2007. 
instrument de recherche adopté dans ces domaines est le recueil de témoignages oraux auprès d'individus et de familles ayant directement vécus la Partition. Le travail d'Urvashi Butalia constitue à cet égard une référence incontournable. Elle présente une collection d'entretiens semi-directifs qu'elle a réalisés auprès d'environ soixantedix personnes sur plusieurs années. Son objectif n'est pas tant d'étudier l'histoire de la Partition que d'analyser la mémoire qui en subsiste chez ceux qui l'ont vécue. Elle expose les voix de ceux qui ont toujours été tenus à l'écart du discours officiel sur la Partition. Elle nous apprend ainsi que s'il n'y a pas à proprement parler de mémoire collective publique de la Partition, il existe en revanche de multiples mémoires individuelles qui sont perpétuées à travers des rituels de commémoration privés et la transmission d'histoires individuelles au sein des familles. Cet usage des récits oraux n'est toutefois pas sans écueil. Si Butalia sait qu'il n'y a pas de mémoire pure et que tout récit oral est à sens unique, il apparaît néanmoins que son étude manque parfois de réflexivité. Le problème est de savoir quelle volonté ces efforts d'archivage et d'écriture de l'histoire reflètent in fine, celle de l'interviewer ou celle de l'interviewé. Il existe un décalage relatif, que seules de rigoureuses précautions méthodologiques peuvent parvenir à combler, entre la manière dont les interviewés décrivent leur histoire et leur identité, et la manière dont celle-ci est représentée par l'interviewer, puisque les premiers n'écrivent que très rarement eux-mêmes leurs témoignages ${ }^{35}$. Pourtant, «le passage de la mémoire à l'histoire requiert de chaque groupe social la redéfinition de son identité à travers la revitalisation de sa propre histoire. La tâche de se souvenir fait de chacun un historien. La demande d'histoire a ainsi largement déplacé le cercle des historiens professionnels ${ }^{36} »$.

En parallèle au recours aux témoignages oraux, on observe une recrudescence de l'usage des ressources littéraires et cinématographiques dans l'analyse de la dimension humaine de la Partition. Quand l'analyse scientifique de la violence était considérée illusoire, et par conséquent exclue du champ de l'histoire, la représentation de la douleur était cantonnée à la littérature. Or, il apparaît aujourd'hui que les références littéraires et cinématographiques, un de ces fragments chers à Pandey, offrent un matériau de grande valeur pour l'historien dans son étude de la dimension humaine de la Partition. Au milieu des années 1990, on assiste à la publication de pas moins de trois anthologies de nouvelles ${ }^{37}$ et à la réédition de nombreux romans ${ }^{38}$ traitant de la Partition. Il est frappant de constater que cet usage de la fiction se retrouve, bien que dans des perspectives légèrement différentes, autant

35 - J.-L. Racine (avec J. Racine), «Beyond Silence : A Dalit Life History in South India», in S. Blackburn et D. Arnold (dir.), Telling Indian Lives : Biography, Autobiographies and Life-Histories in India, New Delhi, Indiana University Press, Bloomington \& Permanent Black, p. 252-280.

36 - P. Nora, Les lieux de mémoire, Paris, Gallimard, 1984.

37 - A. Bhalla (éd.), Stories about the Partition of India, 3 volumes, Delhi, Indus, 1994; S. Cowasjee et K.S. Duggal, Orphans of the Storm : Stories on the Partition of India, New Delhi, UBS Publishers, 1995 ; M. Hasan (éd.), India Partitioned: The Other Face of Freedom, 2 volumes, New Delhi, Roli, 1995.

38 - Entre autres, Amitav Ghosh, The Shadow Lines, Delhi, Ravi Dayal, 1995; Attia Hussain, Sunlight on a Broken Column, New Delhi, Penguin, 1992; Intizar Hussain, Basti, New Delhi, Indus, 1995; Qurratulain Hyder, River of Fire, New Delhi, Kali for Women, 1998; Kamleshwar, Partitions, New Delhi, Penguin, 2001; Bhisham Sahni, Tamas, New Delhi, Penguin, 2001; Bapsi Sidhwa, Ice-Candy Man, New Delhi, Penguin, 1989; Khuswant Singh, Train to Pakistan, New Delhi, Ravi Dayal, 1996

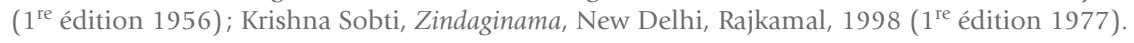


dans l'école de Cambridge que dans les écoles nationalistes et subalternistes. Dans la littérature comme dans le cinéma d'Asie du Sud, la Partition constitue un genre à part entière $^{39}$. Or, loin de se limiter à représenter le passé et l'histoire de la Partition, ces œuvres contribuent à l'émergence d'une mémoire collective des événements de la Partition et mobilisent la mémoire à des fins de construction des communautés à l'échelle locale et nationale ${ }^{40}$. À nouveau cependant, l'usage qui est fait de ces ressources littéraires et cinématographiques apparaît problématique en raison de son manque de réflexivité et de contextualisation. L'usage fait par Ian Talbot de la littérature de la Partition ${ }^{41}$ repose sur une équivalence discutable entre le récit littéraire et l'expérience humaine. Il considère en effet le récit littéraire comme un dérivé de l'expérience personnelle et adopte ainsi une perspective totalisante qui empêche toute utilisation heuristique du corpus littéraire. Chez Pandey, qui se garde de toute prétention totalisante grâce à sa perspective fragmentaire, ce qui pose problème est l'usage quasiment littéral qu'il fait des textes, au point d'en oublier le contexte ${ }^{42}$. Or, seule l'analyse de l'interaction entre le texte et le contexte peut autoriser un usage scientifique rigoureux de la littérature de la Partition. Le recours à ces œuvres de fiction ne peut en aucun cas être considéré comme réflectif et métaphorique, ce qui est le cas de Talbot comme de Pandey, mais doit être au contraire appréhendé comme diffracté et métonymique ${ }^{43}$.

\section{L'oubli comme vertu en Inde et au Pakistan ou comment fuir sa propre responsabilité}

Est-ce parce que les fantômes de la Partition doivent être laissés dans l'oubli et non exhumés pour de fréquents post mortem que le silence s'est imposé ${ }^{44}$ ? Il semble que l'amnésie collective relative à la Partition qui préside en Inde et au Pakistan ait trouvé un terrain fertile dans la tradition propre au sous-continent de ne pas célébrer les événements douloureux de l'histoire. Déterrer les souvenirs refoulés des

39 - I. Bhaskar, «The Persistence of Memory : Historical Trauma and Imagining the Community in Indian Cinema ", communication à la conférence internationale Film Discussion : Peace \& Conflict : Images from Cinema in India and Pakistan, Fifth Annual WISCOMP Conflict Transformation Worshop, New Delhi, 31 octobre 2006. Bien qu'Ira Bhaskar déplore que les cinéastes indiens ne se soient pas saisis de la Partition en tant que thème privilégié, il existe toutefois un certain nombre de films abordant ces événements soit frontalement, soit en trame de fond. Ainsi, au Pakistan : Janat ki Talash de Hasan Askari (1999), Jinnah de Jamil Dehlavi (1999), Khak aur Koon de Masud Pervaiz (1979), Khamosh Pani de Sabiha Sumar (2003), Khartar Singh de Saifudin Saif (1959), Lakhon Mein Ek de Raza Mir (1967); et en Inde : Pinjar; Earth 1947 de Deepah Mehta (2003), Train to Pakistan de Pamela Rook (1997), Dharamputra de B. R. Chopra (1961), Garam Hawa de M.S. Sathyu (1973), Mammo de Shyan Benegal (1986), Refugee de J. P. Dutta (2000), Hey Ram de Kamal Hasan (2000), Meghe Dhaka Tara de Ritwik Ghatak (1960).

40 - S. Malik, «Partition Cinema : Shared History, Differential Perceptions», South Asian Journal, juillet-septembre 2007, p. 137.

41 - I. Talbot, Freedom's Cry: The Popular Dimension in the Pakistan Movement and Partition Experience in North-West India, Karachi, Oxford University Press, 1996, p. 105-123.

42 - G. Pandey, "The Prose of Otherness...», art. cit., 1994, p. 213-221. Voir aussi du même auteur, «In Defense of the Fragment... », art. cit., 1992, p. 47-51.

43 - J. Didur, Unsettling Partition..., op. cit., 2007, p. 42-67.

44 - M. Hasan, "Memories of a Fragmented Nation : Rewriting the Histories of India's Partition", in S. Settar et I. B. Gupta, Pangs of Partition: The Human Dimension, vol. 2, Delhi, Manohar, 2002, p. 175. 
massacres de la Partition présenterait le danger de rouvrir d'anciennes blessures. Comme le souligne l'écrivaine indienne Krishna Sobti, si la Partition est difficile à oublier, il serait également dangereux de s'en souvenir ${ }^{45}$. Urvashi Butalia s'interrogea aussi, quelques années après avoir publié son ouvrage sur la Partition, sur la possibilité de traiter de la violence sans exacerber les tensions ${ }^{46}$. Par ailleurs, il serait, selon Javed Alam, inutile d'étudier ces violences dans la mesure où ni l'État ni aucune organisation d'envergure n'y auraient pris part et que, par conséquent, on ne pourrait en parler comme d'un phénomène général ${ }^{47}$.

Contrairement à l'Allemagne contemporaine dont la culpabilité à l'égard de l'Holocauste et la nécessité éthique de se remémorer les souffrances imposées justifient le devoir de mémoire, Javed Alam et Suresh Sharma affirment que l'oubli des violences de la Partition en Inde est légitimé par la nécessité de préserver l'unité de la nation. Ils considèrent qu'il serait moralement indéfendable de demander aux témoins de la Partition de raconter leurs souvenirs traumatisants, alors que les massacres de la Partition ne seraient plus pour les nouvelles générations qu'un événement historique distant et sans signification pour le présent. L'oubli serait donc bénéfique pour le futur de la vie politique et sociale et des interactions intercommunautaires en Inde. «Le quotidien de la vie retrouve sa normalité si cet événement est oubliée ${ }^{48}$.» Ils concluent qu'il serait donc préférable de mettre l'accent soit sur les manifestations de bonté et d'entraide qui ont aussi eu lieu durant la Partition, soit sur le caractère éminemment tolérant et composite de la civilisation indienne, ce dernier pouvant être mis à mal par l'exhumation des mémoires douloureuses des souffrances de la Partition.

Le présent serait miné par sa servitude à l'égard du passé, une servitude imputée à l'histoire considérée comme un obstacle à la marche du futur. Il serait donc nécessaire d'oublier le passé, de le laisser derrière afin de pouvoir continuer à évoluer. Gandhi, du reste, avait adopté cette thèse: «Je crois dans le proverbe selon lequel une nation heureuse est une nation qui n'a pas d'histoire. Ma théorie personnelle est que nos ancêtres hindous avaient résolu la question pour nous en ignorant l'histoire telle qu'elle est envisagée aujourd'hui et en construisant leur structure philosophique sur des événements moins graves ${ }^{49}$.» Insidieuse, cette conception des dangers de la

45 - A. Bhalla, Partition Dialogues : Memories of a Lost Home, New Delhi, Oxford University Press, 2006, p. 136-165.

46 - U. Butalia, «Partition and Memory», Seminar, n 497, 2001, p. 92-95.

47 - J. Alam, «Remembering Partition : A Dialogue with Suresh Sharma», Seminar, n 461, 1998, p. 99. Si les États indiens et pakistanais n'ont en effet pas directement participé aux massacres de la Partition, il n'en demeure pas moins que ceux-ci furent bel et bien organisés, et non pas spontanés comme on l'entend souvent, et que de nombreux groupes paramilitaires contenant d'ex-militaires les ont dirigés avec l'acquiescement des administrations civiles provinciales. Mais surtout, c'est précisément par leur absence que les États indiens et pakistanais se sont montrés coupables. Dans les provinces à minorités musulmanes telles que les Provinces unies (aujourd'hui l'Uttar Pradesh), la violence a pu être contenue grâce à l'intervention de l'État, pas seulement central mais aussi provincial. Voir P. Verdee, "Partition and the Absence of Communal Violence in Malerkotla» et Y. Khan, "Out of Control? Partition Violence and the State in Uttar Pradesh», in I. Talbot, The Deadly Embrace : Religion, Politics and Violence in India and Pakistan 1947-2002, Karachi, Oxford University Press, 2007, respectivement p. 16-36 et p. 36-60.

48 - J. Alam, "Remembering Partition : A Dialogue with Suresh Sharma», art. cit., 1998, p. 100.

49 - Cité dans S. Khilnani, "Gandhi and History», Seminar, n 461, 1998, p. 110. 
mémoire a pénétré l'histoire académique, comme en témoigne cette adresse présidentielle lors du Congrès de l'histoire indienne en 1964 : «l'histoire ne doit pas faire revenir à la mémoire les horribles aberrations de la nature humaine, les crimes lâches et honteux, les divisions et les conflits, les dégénérations et le déclin, mais au contraire exalter les plus hautes valeurs de la vie, les traditions de la culture et les nobles actes de sacrifice et de dévotion au service de l'humanité [...]. La raison de cette omission est que de telles choses ressuscitent des tendances malsaines qui militent contre le cours de la solidarité nationale ou de la paix internationale ${ }^{50}$ ». On lit dans un éditorial du Times of India traitant de l'obsession commémorative des attentats du 11 septembre 2001 aux États-Unis et daté du même jour six ans après : «La tradition indienne est différente [de la tradition occidentale en matière de travail de mémoire]. Il y a peu de commémorations des anniversaires négatifs. Prenez la Partition. Elle a généré des ravages dans le sous-continent, des millions de gens périrent durant les émeutes. Se souvenir de cette perte année après année reviendrait seulement à prolonger la haine entre les deux voisins et à contaminer les sentiments populaires fraternels, qui agissent comme un frein sur les deux États belligérants, entre les uns et les autres. Au lieu de se remémorer les émeutes, à minuit, nous célébrons notre Indépendance. » Dans la même veine: "Lorsqu'on lui demande de se souvenir de la Partition, Ramji, âgé de 84 ans, vous demande de parler plutôt du présent. "Oubliez le passé; Dieu nous en garde, personne ne devrait vivre de telles époques. Ce fut des temps sanglants et je n'ai pas le courage de m'en souvenir. Je prie pour que cela n'arrive plus jamais" ${ }^{\prime 1}$." Il semble qu'en réalité, ce danger associé à l'idée de ressusciter le passé corresponde plutôt à une gêne liée à un profond sentiment de culpabilité. La frontière qui sépare la victime du coupable durant les massacres de la Partition est extrêmement poreuse. Il n'y a pas de victimes pures durant les massacres de la Partition. Dans nombre de familles, certains sont morts et d'autres ont tué, d'où l'impossibilité de se poser en tant que victime ou martyr. Le cas de la communauté sikh, dont l'ethos est profondément marqué par les notions d'héroïsme et de martyre, est ici exemplaire. Le processus de mémorialisation est bloqué d'une part à cause des humiliations subies durant la Partition et, d'autre part, car les sikhs n'étaient pas seulement victimes mais aussi bourreaux sans héroïsme qui furent les principaux assaillants au Pendjab oriental ${ }^{52}$. Ainsi, une condition nécessaire pour un travail de mémoire heuristique serait de reconnaître au préalable sa propre culpabilité, ce qui ni les États ni les populations ne sont prêts à faire.

\section{La mémoire comme catharsis}

\section{ou comment faire la paix avec la Partition}

La mémoire, bien plus que l'oubli, paraît à même de produire une catharsis collective et de promouvoir la réconciliation entre les populations et les gouvernements indiens et pakistanais. Le silence n'est pas synonyme d'oubli. Le traumatisme de la

50 - Cité dans R. Bhargava, «History, Nation and Community : Reflections on Nationalist Historiography of India and Pakistan ", Economic and Political Weekly, vol. 35 (4), 22-28 janvier 2000, p. 195.

51 - R. Bhatia, "Sixty Years On, Ghosts of Partition Gone but Colony near Kotla Craves for Sanction», The Indian Express, 15 août 2007, p. 1.

52 - P. Brass, «Victims, Heroes or Martyrs? Partition and the Problem of Memorialization in Contemporary Sikh History», Sikh Formations, vol. 2, n 1, juin 2006, p. 21-22. 
Partition peut être maintenu sous silence mais pas éradiqué de la mémoire collective. S'il peut certes être dangereux de faire resurgir les souvenirs de la Partition, il nous semble bien plus pernicieux de les laisser être mobilisés à des fins politiques et belliqueuses. Le déni d'histoire ne peut en aucun cas permettre d'établir une identité stable et de construire un futur pacifié. Seule une volonté commune de dépasser les anciennes querelles, à travers une analyse introspective sincère et rigoureuse, peut permettre de désactiver les dynamiques conflictuelles qui se nourrissent des mémoires douloureuses. L'écrivain Manohar Shyam Joshi s'insurge ainsi de ce qu'il considère comme l'un des principaux problèmes de l'Inde, à savoir qu'il $\mathrm{s}^{\prime}$ agit «d'une nation dévolue à l'oubli plutôt qu'au souvenir » ${ }^{53}$. Il poursuit : "Nous existons soit dans la réalité présente soit dans l'infini. Dans nos shhradhs [commémorations religieuses faites d'offrandes aux âmes des ancêtres afin qu'elles soient révérées], nous nous souvenons seulement de trois générations. " De même, l'acteur de théâtre Habib Tanvir et le peintre Ram Kumar considèrent que seul l'art peut permettre d'exorciser les effets néfastes de la Partition, d'en désactiver l'héritage de haine $^{54}$. «En dépit de décennies de suspicions et d'antipathies mutuelles qui ont conduit à une course à l'armement inconsidérée et ont contribué à l'arriération et à l'immense pauvreté de la région, il est encore possible pour les populations, plutôt que les gouvernements, de faire sens du poignant travail des écrivains et des poètes et de réfléchir sur comment et pourquoi une génération a été prise dans la ligne de feu de la bigoterie religieuse, de l'intolérance et du sectarisme ${ }^{55}$. " Dans cette perspective, l'objectif initial ne doit pas nécessairement être la paix, mais de plus larges interactions et une meilleure compréhension de l'autre. De nombreux professionnels et des groupes engagés travaillant dans leur propre domaine - média, art, théâtre, film, danse - sont ainsi déjà en contact avec d'autres professionnels et des groupes similaires de l'autre côté de la frontière, pour discuter et collaborer sur des questions allant de l'éducation, ou de la violence dans la société, la violence domestique ou envers les femmes, au fondamentalisme religieux, aux enjeux légaux, aux armes nucléaires, à l'environnement, et au travail ${ }^{56}$.

Ainsi, en parallèle à l'établissement des faits pouvant servir de base à une reconnaissance publique - ce qui est en particulier la tâche de l'historien - et à la mise en œuvre d'un travail de mémoire subjectif, à la fois au niveau individuel et au niveau collectif, afin d'organiser les mémoires et de leur conférer un sens, le régime du merveilleux et des arts est essentiel pour permettre que ce qui s'est passé ne soit pas oublié et, plus encore, ne se reproduise pas à nouveau ${ }^{57}$. À cet égard, l'analyse de diverses initiatives fondées sur ce régime de vérité aide à identifier des processus susceptibles de favoriser la réconciliation, c'est-à-dire la possibilité de partager un présent qui ne soit pas la reproduction du passé.

53 - P. Philiphose, «Sixty Years of Remembering», The Indian Express, 13 août 2007, p. 10.

54 - Ibid., p. 10.

55 - M. Hasan, "Memories of a Fragmented Nation...», art. cit., 2002, p. 176.

56 - B. Sarwar, «Women's Role in Building Peace between India and Pakistan », Background Paper, Montréal, McGill University, 30 juillet- ${ }^{\text {er }}$ août 2004, p. 1-32, disponible sur http://www.mcgill.ca/ files/mcrtw/BeenaSarwarpaper.pdf.

57 - B. Pouligny, «Mémoires et violences...», art. cit., 2002, p. 3. 
Les commémorations officielles fournissent une occasion privilégiée d'étudier les modalités du travail de mémoire, ou au contraire d'oubli. À l'occasion du soixantième anniversaire de l'indépendance et de la Partition, il fut frappant de constater que ni le Premier ministre indien ni le Premier ministre pakistanais n'utilisaient le terme "Partition» dans leurs discours respectifs adressés aux nations indienne et pakistanaise. Une minute de silence a néanmoins été respectée au Pakistan à la mémoire des centaines de milliers de personnes mortes durant la Partition. Le traitement réservé à la Partition dans les quotidiens majeurs en Inde et au Pakistan est éminemment divergent. En Inde, la commémoration de la Partition a été presque totalement occultée au profit de la célébration de l'indépendance, qui a surtout été l'occasion d'affirmer les réussites de l'Inde contemporaine et la foi dans le futur. Au Pakistan, la Partition a trouvé un écho plus large dans la mesure où elle est considérée comme la condition indispensable de la création du Pakistan, mais aussi en raison des incertitudes qui entourent la trajectoire passée et le devenir de l'identité nationale pakistanaise.

À l'occasion de la célébration du soixantième anniversaire de la création du Pakistan et de la Partition, une initiative extrêmement importante, au croisement des problématiques du devoir d'histoire, du travail de mémoire, et de leurs relations avec l'identité nationale, s'est tenue au Conseil national des arts du Pakistan (National Arts Council of Pakistan, NACP) à Karachi, du 11 au 14 août 2007. Intitulée «The Shanaakht [identité] Project", elle a été lancée par les Archives citoyennes du Pakistan (Citizen Archives of Pakistan, CAP), une organisation non gouvernementale créée par de jeunes pakistanais - ils ont entre 20 et 30 ans - et dédiée à la commémoration des expériences individuelles et collectives de toutes les couches sociales et de toutes les communautés du Pakistan liées à la création du pays. La présidente des Archives citoyennes du Pakistan, Sharmeen Obaid-Chinoy, journaliste et documentariste, explique : "C'est la première initiative privée montée et dirigée par de jeunes gens afin de collecter, archiver, étudier, disséminer et exposer tous les aspects de l'histoire pakistanaise à la fois avant et après la Partition. " Elle ajoute: "Nous sommes un peuple perdu, sans histoire ni mémoire du passé. La Partition de 1947 fut une expérience traumatisante et s'en souvenir nous informe sur la manière dont le Pakistan a été créé et sur l'identité des personnes qui en sont à l'origine. " Interroger l'identité pakistanaise contemporaine à partir de son passé afin d'envisager un meilleur futur, telle était l'idée de ce festival multimédia qui réunissait à l'attention du grand public des expositions de photographies et de peintures, des documentaires, des pièces de théâtre, des sessions de contes, mais qui invitait aussi chaque personne présente et désireuse d'enregistrer son expérience, à la raconter dans l'intimité de l'isoloir d'une sorte de photomaton équipé d'une caméra. "Le but ultime de cette initiative est de pouvoir ouvrir, dans un avenir plus ou moins proche, un musée en libre accès comprenant une librairie, une salle d'exposition exposant des archives, des œuvres d'art, des photographies, des vidéos, etc. », conclut sa présidente.

La troupe de théâtre Ajoka, créée en 1984 à Lahore et dirigée par les époux Madeeha Gauhar, metteuse en scène, et Shahid Nadeem, scénariste, a fait de la Partition la principale source de son inspiration et a d'emblée conçu sa production artistique comme un vecteur en faveur du changement social et politique et de l'instauration de la paix au Pakistan, ainsi qu'entre ce dernier et l'Inde. On compte, à son actif, 
cinq pièces traitant, plus ou moins directement, de la Partition, qui sont autant d'actes de paix visant à faire émerger une mémoire collective commune: Aik Thee Nani (Il était une fois une grand-mère), Dhuk Darya (La rivière de la souffrance), Toba Tek Singh (il s'agit du nom d'un village du Pendjab pakistanais et du titre éponyme d'une célèbre nouvelle de l'écrivain pakistanais Sadat Hasan Manto), Shehr-e-Afsos (La ville du chagrin) et Border-Border (Frontière-Frontière).

L'une des principales réussites de cette troupe en matière de rapprochement entre l'Inde et le Pakistan consiste en la mise en place d'un réseau d'artistes provenant des deux Pendjab, le All Punjab Performing Artists Network (APPAN, qui signifie «nousmêmes» en Punjabi). Selon Madeeha Gauhar : "Ce réseau repose sur l'idée que la construction de la paix, la promotion d'idées partagées et d'un héritage culturel commun, sont des tâches qui ne peuvent être laissées aux institutions gouvernementales mais qui doivent être entreprises par les intéressés eux-mêmes, c'est-à-dire les populations. » "Ce réseau permet donc de renforcer les liens entre les artistes des deux Pendjab et de fournir une plateforme commune à partir de laquelle ils peuvent partager leurs expériences, collaborer et organiser des événements communs tels que des ateliers, des pièces de théâtre, à l'occasion notamment des anniversaires de poètes sufis, de héros du mouvement d'indépendance, de festivals religieux, etc. " C'est par le biais d'un festival de théâtre indo-pakistanais, rebaptisé lors de sa seconde édition en 2005 «Panj Pani» (Les cinq rivières), que ce réseau a pu voir le jour. Le festival a depuis été organisé chaque année. Il est intéressant de noter qu'alors que l'édition 2005 du festival n'avait pas de thème, celle de 2006, qui n'en avait pas non plus au départ, fut intitulée au dernier moment Beyond Borders (Au-delà des frontières). Ce thème a été attribué a posteriori, après réception des propositions de pièces, parce que plus d'un tiers des spectacles proposés abordaient de près ou de loin le thème de la Partition ou des sujets y étant liés tels que la migration, l'identité, la frontière, tandis que les autres mettaient en avant la culture commune entre les deux pays. L'édition 2007 du festival, qui s'est déroulée à Lahore du 31 mars au 7 avril, avait quant à elle pour thème : Reinterpreting History (Réinterpréter l'histoire).

\section{Conclusion}

Si le but de la Partition du Raj britannique était de pacifier la région, force est alors de constater que, soixante ans après, cela se révèle être un relatif échec. D'un point de vue interétatique, la création de l'Inde et du Pakistan est loin d'avoir stabilisé l'environnement régional sud-asiatique. Ces deux États se sont affrontés militairement à quatre reprises et, depuis leurs essais nucléaires respectifs en 1998, la menace d'une guerre nucléaire est une réalité incontournable. En Inde, d'un point de vue de politique intérieure, loin d'avoir mis fin aux conflits intercommunautaires entre hindous et musulmans, la mémoire de la Partition continue, au contraire, à les alimenter. L'idéologie du Pakistan, qui place l'Islam au cœur de l'identité nationale pakistanaise, s'est vue contredite d'abord par la sécession du Bangladesh, puis par l'essor des conflits ethniques et sectaires. Ainsi, la Partition serait surtout la source d'un paradigme politique conflictuel qui régit aussi bien les relations entre l'Inde et le Pakistan que celles que ces deux pays entretiennent avec 
leurs populations, en particulier les minorités ${ }^{58}$. Seul un authentique processus de reconnaissance des faits et des responsabilités, réalisé simultanément en Inde et au Pakistan, pourrait permettre de conduire à la réconciliation. Universitaires, intellectuels, artistes et militants peuvent jouer un rôle considérable dans l'initiation d'un tel processus. En offrant une histoire objective de la dimension humaine de la Partition, en proposant la création d'un mémorial dédié à la mémoire des victimes de la Partition au poste-frontière de Wagah-Attari ${ }^{59}$, en animant des débats rassemblant les populations des deux pays, en réalisant des œuvres d'art fondées sur l'histoire de la Partition de part et d'autre de la frontière, bref en forgeant de telles traces du passé dans la mémoire collective, ils peuvent agir sur les mentalités et contraindre les responsables politiques et les diplomates à se saisir de la mémoire de la Partition. Car il est évident que toutes ces initiatives se révèleront inutiles sans une véritable volonté politique de chaque côté de la frontière. Ce n'est qu'à travers la représentation au présent de ce passé absent qui ne passe pas qu'une "mémoire apaisée» pourra voir le jour ${ }^{60}$. Et ce n'est finalement qu'au sein du terreau d'une telle mémoire réconciliée que l'Inde et le Pakistan pourront voir des identités nationales stables et tolérantes éclore et s'épanouir.

58 - I. Ahmed, «The 1947 Partition of India : A Paradigm for Pathological Politics in India and Pakistan", Asian Ethnicity, vol. 3, n 1, 2002.

59 - Un projet, initié par l'universitaire Ishtiaq Ahmed et intitulé Memorandum for Peace and Friendship Memorial at Wagah-Attari Border, a proposé la construction d'un mémorial dédié à la mémoire des victimes de la Partition, sur le site du poste-frontière indo-pakistanais au Pendjab. Cependant, ce projet, qui a été d'abord soumis en 1998, puis relancé en 2002 et 2004, n'a pour l'instant reçu aucun soutien officiel de la part des gouvernements indiens et pakistanais. Voir Association for Communal Harmony in Asia (ACHA), Peace Bulletin, vol. 7 (10), 6 octobre, 2004.

60 - P. Ricœur, La mémoire, l'histoire, l'oubli, Paris, Seuil, 2000, p. 645. 